Pacific Journal of Mathematics

ON ARC LENGTH SHARPENING S

W hun ANDREw EtTung 


\title{
ON ARC LENGTH SHARPENINGS
}

\author{
William A. EtTLing
}

\section{This paper introduces two new sharpenings:}

ThEOREM. Let $A$ denote a rectifiable arc (with length $l(A))$ of a metric space, let $P$ denote a finite, normally-ordered subset of $A$, and let $l\left(T^{*}(P)\right)$ denote the linear content of a

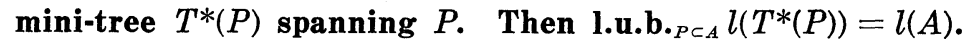

Definition. If $E$ is a nonempty subset of a set $P$ that is spanned by tree $T$, then $T$ is said to be on $E$.

THEOREM. Let $\sigma(E)$ denote the greatest lower bound of the linear contents of all trees on $E$. If $A$ denotes a rectifiable arc of a finitely compact metric space, then l.u.b. . $\subset A_{A} \sigma(E)=l(A)$, where $E$ denotes any finite normallyordered subset of $A$.

On arc length sharpenings. ${ }^{1}$ It is convenient to call an unordered pair of distinct points $p, q$ of a metric space $M$ a segment, denoted by $\{p, q\}$. Each of the points $p, q$ of the segment $\{p, q\}$ is an endpoint of the segment, and the length of $\{p, q\}$ is the distance $p q$ of its endpoints.

A nonempty set $S$ of distinct segments forms a chain $C$ provided the end points of the segments may be labelled $a_{0}, a_{1}, \cdots, a_{k}$ (with all the $a_{i}$ 's representing pairwise distinct elements of $M$ ) so that the elements of $S$ are $\left\{a_{0}, a_{1}\right\},\left\{a_{1}, a_{2}\right\}, \cdots,\left\{a_{k-1}, a_{k}\right\}$. The chain is said to join $a_{0}$ and $a_{k}$; the points $a_{0}, a_{1}, \cdots, a_{k}$ are the vertices of the chain.

A nonempty set $S$ of segments forms a tree $T$ provided each two distinct points of the set of endpoints of the segments are joined by exactly one chain of its segments. The vertices of $T$ are the endpoints of its segments. The segments of a tree are called branches, and the linear content of a tree is the sum of the lengths of its branches. If a tree $T$ has set $E$ as its vertex set, then $T$ is said to span $E$. If $E$ is a nonempty subset of a set $P$, and tree $T$ spans $P$, then $T$ is said to be on $E$.

A finite subset $E$ (containing at least two points) of $M$ is spanned by only a finite number of trees. Let $L(E)$ denote the minimum of the linear contents of the trees that span $E$ and let $T^{*}(E)$ symbolize any tree spanning $E$ whose linear content $l\left(T^{*}(E)\right)$ equals $L(E)$. $T^{*}(E)$ is referred to as a mini-tree spanning $E$.

Denote by $\sigma(E)$ the greatest lower bound of linear contents of all trees that span $P$ where $P \supset E(P$ is a finite subset of $M)$; that

\footnotetext{
1 From research for University of Missouri Dissertation (1973).
} 
is, $\sigma(E)$ is the greatest lower bound of the linear contents of all trees on $E$. Clearly $0<\sigma(E) \leqq L(E)$, and easy examples of subsets $E$ of the Euclidean plane exist such that $\sigma(E)<L(E)$.

A subset $A$ of a metric space is an arc provided it is the homeomorph of a line segment: that is, $A=f(I)$, where $f$ is biuniform and bicontinuous in the line segment $I=[a, b], a<b$. The points $\alpha=f(a), \beta=f(b)$ are the endpoints of $A$. Calling $\alpha$ the initial point and $\beta$ the terminal point of $A$ serves to orient the arc, and a finite subset $P=\left(\alpha_{1}, \alpha_{2}, \cdots, \alpha_{n}\right)$ of $A$ is normally ordered provided these points are encountered in the order of their subscripts when the arc is traversed from $\alpha$ to $\beta$.

This paper furnishes the following two primary theorems, along with several other results in support of these theorems.

Theorem 1. For each rectifiable arc $A$ of a metric space,

$$
\underset{P \subset A}{\text { l.u.b. }} l\left(T^{*}(P)\right)=l(A),
$$

where $P$ represents a finite normally ordered subset of $A$.

Theorem 2. If A. denotes a rectifiable arc of a finitely compact metric space $M$, with length $l(A)$, then

$$
\text { l.u.b. } \sigma(E)=l(A),
$$

where $E$ denotes any finite normally ordered subset of $A$.

The literature refers to this kind of theorem as an arc length "sharpening." Theorem 1 is similar in nature to a "second sharpening" as presented by Blumenthal [1], and Theorem 2 partially answers the question of a possible "third sharpening" posed by the same author. Menger and Mimura [3] have previously proved a third sharpening for arcs of Euclidean space.

For arcs of the type of space prescribed by Theorem 2, points not on the arc may be allowed to enter into the computation of its length.

If $p_{i}$ is a vertex of a tree $T$, let $B\left(p_{i}\right)$ denote the set of vertices of $T$, which paired with $p_{i}$, form branches of $T$. Call $B\left(p_{i}\right)$ the set of vertices of $T$ sending branches to $p_{i}$. The cardinality of $B\left(p_{i}\right)$ is denoted by $o\left(p_{i}\right)$ and is called the order of $p_{i}$. A subtree of a tree $T$ is a tree which has each of its branches a branch of $T$.

Lemma 1. Let $T$ be a tree spanning vertex set $E=\left\{p_{1}, p_{2}, \cdots, p_{n}\right\}$. If $p_{i} \in E$ has order $o\left(p_{i}\right)=k$, then $E-\left\{p_{i}\right\}$ is the union of $k$ non- 
empty mutually exclusive subsets, each of which is a vertex set of a subtree of $T$ or is a single point.

Proof. To say that a vertex $p_{i}$ of a tree $T$ is of order $k$ means that $p_{i}$ is joined directly (paired with) exactly $k$ other vertices of $T$ by branches of $T$. So with respect to pairs of points of $E$ which are branches of $T$, there are $k$ distinct disjoint subsets of $E$ which are joined only through point $p_{i}$ by branches of $T$. For if the contrary be assumed, some two of them would have a vertex in common and the two would be connected by branches of $T$. Thus, in the tree $T$, there would exist two sequences of branches joining any point of the two subsets with $p_{i}$, contradicting the uniqueness of chains in a tree.

THEOREM A. If $T$ is a tree spanning a set $E$ of $n$ distinct points $(n \geqq 2)$, then $T$ has exactly $n-1$ branches.

Proof. The statement is clearly valid for $n=2$. Suppose the result holds for trees with vertex sets $E$ of cardinality at most $n-1$, it will be shown valid for $n$. Let $E$ be a set of $n$ distinct points, $T$ a tree spanning $E$, and $p \in E$ a vertex of $T$ of order $r$. By Lemma 1, the set $E-\{p\}$ is the sum of a finite number of subsets $E_{1}, E_{2}, \cdots, E_{r}$, each of which admits a subtree $T_{i}$ of $T$ but ceases to do so after the adjunction of any point of $E-\{p\}$ not belonging to it, or is a single point. If $E_{i}$ consists of $n_{i}$ points, then $n_{i} \leqq n-1(i=1,2, \cdots, r)$. By the inductive hypothesis, if $E_{i}$ admits a subtree $T_{i}$, then $T_{i}$ consists of exactly $n_{i}-1$ branches. If $E_{i}$ consists of a single point, no branch of $T$ is admitted by $E_{i}$. In either case, each $E_{i}$ admits exactly $n_{i}-1$ branches of $T$. Hence $E-\{p\}$ admits exactly

$$
\sum_{i=1}^{r}\left(n_{i}-1\right)=\sum_{i=1}^{r} n_{i}-r=(n-1)-r
$$

branches of $T$. Since $T$ is a tree, there is exactly one point $p_{i} \in E_{i}$ such that $\left\{p_{i}, p\right\}$ is a branch of $T(i=1,2, \cdots, r)$. Hence $T$ has exactly $((n-1)-r)+r=n-1$ branches.

A normally ordered subset $P=\left\{p_{1}, p_{2}, \cdots, p_{n}\right\}$ of an arc $A$ with endpoints $a^{\prime}, b^{\prime}$ is a homogeneous $\varepsilon$-chain provided

(1) $a^{\prime} p_{1}<\varepsilon, p_{n} b^{\prime}<\varepsilon$

(2) $p_{i} p_{j}=\varepsilon$, for $|i-j|=1 \quad(i, j=1,2, \cdots, n)$,

(3) $p_{i} p_{j} \geqq \varepsilon$, for $|i-j|>1 \quad(i, j=1,2, \cdots, n)$.

If a normally ordered subset is such that $a^{\prime} p_{1}<\varepsilon, p_{n} b^{\prime}<\varepsilon, p_{i} p_{i+1} \leqq \varepsilon$ for $i=1,2, \cdots, n-1$, then $P$ is called an $\varepsilon$-chain. 
Three known lemmas needed in the proof of Theorem 1 will now be stated without proof (cf. [1]).

Lemma 2. For each positive number $\varepsilon$, there exists a homogeneous e-chain in $A$.

LEMma 3. For every positive number $\delta$ there exists a positive number $\varepsilon$ such that every $\varepsilon$-chain in $A$ is $\delta$-dense in $A$.

LemMa 4. Let $A=f(I)$ be a rectifiable arc of a metric space with length $l(A)$. Then any positive number $\eta$ implies the existence of a positive $\delta$ such that for each finite normally ordered subset $P$ of $A$ which is $\delta$-dense in $A, l(P)>l(A)-\eta$.

Proof of Theorem 1. Since $l\left(T^{*}(P)\right) \leqq l(P)$, for $P \subset A$, then

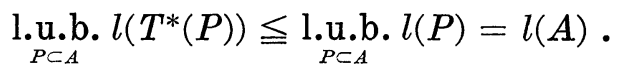

It remains to show that l.u.b. ${ }_{P \subset A} l\left(T^{*}(P)\right) \geqq l(A)$. By Lemma 4, $\eta>0$ implies the existence of a positive $\delta$ such that for each $P$ which is $\delta$-dense in $A, l(P)>l(A)-\eta$. By Lemma 3, there corresponds to this $\delta$ a positive $\varepsilon$ such that every $\varepsilon$-chain in $A$ is $\delta$ dense in $A$, and Lemma 2, there exists for this $\varepsilon$ a homogeneous $\varepsilon$-chain $P=\left\{p_{1}, p_{2}, \cdots, p_{n}\right\}$ in $A$. Then $P$ is $\delta$-dense in $A$ and $l(P)>$ $l(A)-\eta$. Now $P$ being a normally ordered set and a homogeneous $\varepsilon$-chain imply that $l(P)=(n-1) \varepsilon$. Since each two points of $P$ has distance at least $\varepsilon$ and tree $T^{*}$ spanning $P$ has $n-1$ branches, $l\left(T^{*}(P)\right) \geqq(n-1) \varepsilon$. So for each homogeneous $\varepsilon$-chain $P, l(P)=$ $l\left(T^{*}(P)\right)$. Hence corresponding to each $\eta>0$ a subset $P$ of $A$ exists with $l\left(T^{*}(P)\right)>l(A)-\eta$; that is,

$$
\text { l.u.b. } l\left(T^{*}(P)\right) \geqq l(A) \text {. }
$$

The remainder of this paper is presented in support of Theorem 2. The following problem is similar to one found in [2].

Problem $T_{n}$. Given a set $E=\left\{p_{1}, p_{2}, \cdots, p_{n}\right\}$ of $n \geqq 3$ points of a metric space $M$. Find the mini-tree(s) on $E$.

If $p, q, r \in M$ are linear with $q$ metrically between $p$ and $r$, then a mini-tree on set $\{p, q, r\}$ is $\{\{p, q\},\{q, r\}\}$; that is, no additional vertices are required to yield a mini-tree on $\{p, q, r\}$. Further, the trees $\{\{p, r\}\}$ and $\{\{p, q\},\{q, r\}\}$ have the same linear content.

Definition. A tree $T$ is an $R$-tree on a subset $E=\left\{p_{1}, p_{2}, \cdots, p_{n}\right\}$ of $n \geqq 3$ points of $M$ provided the set of all vertices $p_{1}, p_{n}, \cdots, p_{n}$, 
$q_{1}, \cdots, q_{k}$ of $T$ are elements of such that $(1) o\left(q_{i}\right) \geqq 3(i=1,2, \cdots, k)$, and $(2) 0 \leqq k \leqq n-2$.

THEOREM B. Let $E=\left\{p_{1}, p_{2}, \cdots, p_{n}\right\}$ be a finite subset of $n \geqq 3$ points of a metric space $M$. If a solution of the Problem $T_{n}$ exists, then it is an $R$-tree on $E$ or it can be replaced by an $R$-tree having the same linear content.

Proof. The result is trivial, if $k=0$, in which case $o\left(q_{i}\right) \geqq 3$ is vacuously satisfied. So suppose that $k>0$ and that $T$ is a minitree on $E$ spanning $E^{A}=\left\{p_{1}, p_{2}, \cdots, p_{n}, q_{1}, \cdots, q_{k}\right\}$ (augmented vertex set). It is easy to see that $o\left(q_{i}\right)>1(i=1,2, \cdots, k)$. Suppose $o\left(q_{i}\right)=2$ for some $i$ and that $B\left(q_{i}\right)=\left\{r^{\prime}, r^{\prime \prime}\right\} \subset E^{A}$. If $q_{i}$ is linear with vertices $r^{\prime}, r^{\prime \prime}$, then branches $\left\{r^{\prime}, q_{i}\right\},\left\{q_{i}, r^{\prime \prime}\right\}$ can be replaced by $\left\{r^{\prime}, r^{\prime \prime}\right\}$ without increasing the linear content of $T$. On the other hand, if $r^{\prime}$, $r^{\prime \prime}, q_{i}$ are not linear, the triangle inequality implies that $r^{\prime} r^{\prime \prime}<$ $r^{\prime} q_{i}+q_{i} r^{\prime \prime}$. Then the tree formed by replacing branches $\left\{r^{\prime}, q_{i}\right\}$, $\left\{q_{i}, r^{\prime \prime}\right\}$ by $\left\{r^{\prime}, r^{\prime \prime}\right\}$ would decrease the linear content of $T$, contradicting the minimal linear content assumption on $T$. Thus $o\left(q_{i}\right) \geqq 3$ for each $i(i=1,2, \cdots, k)$ or $T$ can be replaced by a tree of equal linear content having this property.

Without loss of generality, it may be assumed that $T$ has $o\left(q_{i}\right) \geqq 3$ for each $i(i=1,2, \cdots, k)$.

Then the number of branches leading from $q$-points is at least $3 k / 2$; the number from $p$-points at least $n / 2$. But $n+k-1=$ (total number of branches of $T) \geqq 3 k / 2+n / 2$, from which it is easily deduced that $k \leqq n-2$. This concludes that proof.

Let $T, T^{\prime}$ be $R$-trees with vertex sets $\left\{p_{1}, p_{2}, \cdots, p_{n}, q_{1}, q_{2}, \cdots, q_{k}\right\}$ and $\left\{p_{1}, p_{2}, \cdots, p_{n}, q_{1}^{\prime}, q_{2}^{\prime}, \cdots, q_{k}^{\prime}\right\}$, respectively. The trees $T$ and $T^{\prime}$ have the same structure provided the mapping $f$ with $f\left(p_{i}\right)=p_{i}, f\left(q_{j}\right)=q_{j}^{\prime}$ $(i=1,2, \cdots, n ; j=1,2, \cdots, k)$ maps $B\left(p_{i}\right)$ onto $B^{\prime}\left(p_{i}\right)$ and $B\left(q_{i}\right)$ onto $B^{\prime}\left(q_{j}\right)$, where $B^{\prime}\left(p_{i}\right)$ and $B^{\prime}\left(q_{j}\right)$ are subsets of the vertex set of $T^{\prime}$.

In general, there will exist infinitely many trees having the same structure. The relation "has the same structure as" on the class of all $R$-trees on a given finite set $E$ is an equivalence relation. Because $0 \leqq k \leqq n-2$ and a structure class is determined largely by the $k$ additional vertices, it is further seen that the number of equivalence classes of $R$-trees on a given set of $n$ points of $M$ under the relation "has the same structure as" is finite. Let these equivalence classes be denoted by $C_{1}, C_{2}, \cdots, C_{N}$.

Theorem C. Let $E=\left\{p_{1}, p_{2}, \cdots, p_{n}\right\}$ be a subset of a finitely compact metric space $M$. There exists a mini-tree on $E$. (As in [2], p. 447, with modifications.) 
Proof. Since there are only a finite number of the aforementioned equivalence classes, it suffices to show that if $C \in\left\{C_{1}, C_{2}, \cdots, C_{N}\right\}$, then there exists on $E$ a mini-tree in $C$.

The structure of trees in $C$ stipulates which of the pairs $q_{i} p_{j}$, $q_{i} q_{j}, p_{i} p_{j}$ will be segments as branches of trees in $C$.

If $k=0$, there are no $q$ points. Since $E$ is finite, there are only a finite number of ways to form pairs of elements of $E$. Hence a minimum length tree can be selected.

If $k \neq 0$, each $R$-tree in $C$ has vertex set consisting of $\left\{p_{1}, p_{2}, \cdots, p_{n}\right\}$ together with $k$ additional $q$-points. Let these be denoted $q_{1}, q_{2}, \cdots, q_{k}$. For $i=1,2, \cdots, k$, let $E_{i}=\left\{p_{i_{1}}, p_{i_{2}}, \cdots, p_{i_{i}}\right\}=$ $\left\{p_{j}: p_{j} \in E\right.$ and $\left\{q_{i}, p_{j}\right\}$ is a branch of trace of trees in $\left.C\right\}$. Let $N_{1}$, $\mathrm{N}_{2}$ be sets of unordered pairs of natural numbers, defined as follows:

$N_{1}=\left\{\{i, j\}:\left\{q_{i}, q_{j}\right\}\right.$ is a branch of trees in $\left.C\right\}$,

$N_{2}=\left\{\{i, j\}:\left\{p_{i}, p_{j}\right\}\right.$ is a branch of trees in $\left.C\right\}$. Then the linear content of a tree in $C$ is

$$
f\left(q_{1}, q_{2}, \cdots, q_{k}\right)=\sum_{i=1}^{k} \sum_{j=1}^{\lambda_{i}} q_{i} p_{i_{j}}+\sum_{\{i, j\} \in N_{1}} q_{i} q_{j}+\sum_{\{i, j\} \in N_{2}} p_{i} p_{j} .
$$

Now suppose that $\left\{\bar{q}_{1}, \bar{q}_{2}, \cdots, \bar{q}_{k}\right\}$ represents a specific (fixed) set of $q$-points. Since $\bar{E}^{A}=\left\{p_{1}, p_{2}, \cdots, p_{n}, \bar{q}_{1}, \bar{q}_{2}, \cdots, \bar{q}_{k}\right\}$ is a finite set, there exists a mini-tree $\bar{T}(k)$ spanning set $\bar{E}^{A}$. Let $\bar{L}_{k}$ denote the linear content of this mini-tree. Let set $X=\left\{r: r \in M\right.$ and $\left.\min _{i} r p_{i} \leqq \bar{L}_{k}\right\}$. Then every $q$-point of a tree of minimal linear content in $C$ is in $X$, for otherwise the length of such a tree would be greater than $\bar{L}_{k}$. Thus if $\left\{q_{1}, q_{2}, \cdots, q_{k}\right\}$ is a set of $q$-points of a mini-tree of $C$, then $\left\{q_{1}, q_{2}, \cdots, q_{k}\right\}$ is an element of the Cartesian product $X^{k}$. Since $X$ is bounded and $M$ is finitely compact, $X$ is compact. Tychonoff's theorem implies that $X^{k}$ is compact. But $f\left(q_{1}, q_{2}, \cdots, q_{k}\right)$ is continuous on $X^{k}$, and so assumes a minimum value on $X^{k}$. This proves the theorem.

Suppose that $A$ denotes a rectifiable arc of a finitely compact metric space $M$. Denote by $l(A)$ the length of arc $A$ and let $P_{i}=$ $\left\{p_{i}^{1}, p_{i}^{2}, \cdots, p_{i}^{n(i)}\right\}(i=1,2, \cdots)$ be an $\varepsilon_{i}$-chain in $\operatorname{arc} A$ and $\lim _{i \rightarrow \infty} \varepsilon_{i}=0$. By the above theorem, there exists a mini-tree $T\left(P_{i}\right)$ spanning a set $P_{i}^{*}$ containing $P_{i}$. Denote by $l\left(T\left(P_{i}\right)\right)$ the linear content of such a tree. Then for each $i, l\left(T\left(P_{i}\right)\right) \leqq \sum_{j=1}^{n(i)-1} p_{i}^{j} p_{i}^{j+1} \leqq l(A)$. Hence l.u.b. ${ }_{i} l\left(T\left(P_{i}\right)\right) \leqq l(A)$.

Lemma 5. If $T\left(P_{i}\right)$ is a mini-tree on an $\varepsilon_{i}$-chain $P_{i}$ of $A$, then each branch of $T\left(P_{i}\right)$ has length at most $\varepsilon_{i}$.

Proof. Suppose that the length of a branch of $T\left(P_{i}\right)$ were greater than $\varepsilon_{i}$. Then that branch could be deleted from $T\left(P_{i}\right)$, 
destroying the connectedness of $T\left(P_{i}\right)$; the connectedness could then be restored by pairing an appropriate two successive points of $P_{i}$ as a branch. This would result in a tree $T^{\prime}\left(P_{i}\right)$ of smaller linear content than that of $T\left(P_{i}\right)$, contradicting the mini-property of $T\left(P_{i}\right)$.

Note the following two possibilities:

Either (1) there exists $\delta>0$ and for infinitely many $i$, point $p_{i} \in P_{i}^{*}$ such that distance $p_{i} A \geqq \delta$,

or

(2) for each $\delta>0$, there exists a positive integer $N$ such that $i>N$ implies $p_{i} A<\delta$, for each $p_{i} \in P_{i}^{*}$.

THEOREM D. Let $A$ be a rectifiable arc (with length $l(A)$ ) of a finitely compact metric space $M$ and let $P_{i}(i=1,2, \cdots)$ be an $\varepsilon_{i}$ chain in arc $A$ and $\lim _{i \rightarrow \infty} \varepsilon_{i}=0$. Then statement (2) above holds.

Proof. Suppose the contrary. Then there exists $\delta>0$ and for infinitely many $i$, a point $p_{i} \in P_{i}^{*}$ such that $p_{i}$ has distance from $A$ greater than or equal to $\delta$. The number of points of $P_{i}^{*}$ which can be connected with $p_{i}$ by a chain of $T\left(P_{i}\right)$ of length at least $\delta / 2$ is at least $2^{\left[\delta /\left(2 \epsilon_{i}\right)\right]}$, since by the above lemma, $T\left(P_{i}\right)$ has at least $\left[(\delta / 2) / \varepsilon_{i}\right]$ branches. Since $P_{i}^{*}$ is connected by branches of $T\left(P_{i}\right)$ and $P_{i}^{*}$ contains $P_{i}$, each of the above mentioned $2^{\left[\delta /\left(2 \varepsilon_{i}\right)\right]}$ points is connected to each of the points of the $\varepsilon_{i}$-chain $P_{i}$ on arc $A$ by a chain of length greater than $\delta / 2$. Since $T\left(P_{i}\right)$ is a tree, these chains have no vertices in common. Thus for infinitely many $i, l\left(T\left(P_{i}\right)\right)>(\delta / 2) \cdot 2^{\left[\delta / 2 \varepsilon_{i}\right]}$, and hence $\lim _{i \rightarrow \infty} l\left(T\left(P_{i}\right)\right)=\infty$. But l.u.b. $._{i} l\left(T\left(P_{i}\right)\right) \leqq l(A)<\infty$.

Intuitively, the above result says that for each $\delta>0$, almost all of the mini-trees $T\left(P_{i}\right)$ are contained within a "tube" of radius $\delta$ about the $\operatorname{arc} A$.

TheOREM E (Continuity). Let $l(T(E))$ denote the linear content of a mini-tree $T(E)$ on $E=\left\{p_{1}, p_{2}, \cdots, p_{n}\right\} \subset M$, and suppose that $E^{*}=\left\{p_{1}, p_{2}, \cdots, p_{n}, q_{1}, q_{2}, \cdots, q_{k}\right\}$ is the vertex set of $T(E)$. Let $\varepsilon>0$ be given and consider points $p_{1}^{\prime}, p_{2}^{\prime}, \cdots, p_{j}^{\prime}$ (for some $j$ such that $1 \leqq$ $j \leqq n)$ with distances $p_{i} p_{i}^{\prime}<\delta=\varepsilon /(3(2 n-3))(i=1,2, \cdots, j)$. Then a mini-tree on $E^{\prime}=\left\{p_{1}^{\prime}, p_{2}^{\prime}, \cdots, p_{j}^{\prime}, p_{j+1}, \cdots, p_{n}\right\}$ has linear content $l\left(T\left(E^{\prime}\right)\right)$ such that $\left|l(T(E))-l\left(T\left(E^{\prime}\right)\right)\right|<\varepsilon$.

Proof. Consider a tree $T^{\prime}$ spanning $\left\{p_{1}^{\prime}, p_{2}^{\prime}, \cdots, p_{j}^{\prime}, p_{j+1}, \cdots, p_{n}\right.$, $q_{1}, q_{2}, \cdots, q_{k}$ and having the same structure as mini-tree $T(E)$. Now $l(T(E))=\sum p_{r} p_{s}+\sum p_{r} q_{s}+\sum q_{r} q_{s}$, with $l\left(T^{\prime}\right)$ possibly differing from $l(T(E))$ only in the distances of the types $p_{r} p_{s}$ and $p_{r} q_{s}$. But

$$
p_{r}^{\prime} p_{s}^{\prime} \leqq p_{r}^{\prime} p_{r}+p_{r} p_{s}+p_{s} p_{s}^{\prime}<p_{r} p_{s}+2 \delta,
$$




$$
\begin{aligned}
& p_{r}^{\prime} p_{s} \leqq p_{r}^{\prime} p_{r}+p_{r} p_{s}<p_{r} p_{s}+\delta, \quad \text { and } \\
& p_{r}^{\prime} q_{s} \leqq p_{r}^{\prime} p_{r}+p_{r} q_{s}<p_{r} q_{s}+\delta
\end{aligned}
$$

In total, there are $n+k-1$ branches in the tree $T^{\prime}$ and $k \leqq n-2$. So there are at most $2 n-3$ branches in $T^{\prime}$. Therefore,

$$
\begin{aligned}
l\left(T^{\prime}\right) & <\sum p_{r} p_{s}+\sum_{i=1}^{2 n-3} 2 \delta+\sum p_{r} q_{s}+\sum_{i=1}^{2 n-3} \delta+\sum q_{r} q_{s} \\
& =\sum p_{r} p_{s}+\sum p_{r} q_{s}+\sum q_{r} q_{s}+\sum_{i=1}^{2 n-3} 3 \delta \\
& =l(T(E))+(2 n-3) \cdot 3 \varepsilon /(3(2 n-3)) \\
& =l(T(E))+\varepsilon .
\end{aligned}
$$

But a mini-tree on $E^{\prime}$ has linear content $l\left(T\left(E^{\prime}\right)\right) \leqq l\left(T^{\prime}\right)<l(T(E))+\varepsilon$. Hence $-\varepsilon<l(T(E))-l\left(T\left(E^{\prime}\right)\right)$.

Now it remains to show that $l(T(E))-l\left(T\left(E^{\prime}\right)\right)<\varepsilon$. Suppose the contrary; that is, that there exists $\varepsilon_{0}>0$ such that for each $\delta>0$ (and in particular, for $\delta=\varepsilon_{0} /(3(2 n-3))$ there are points $p_{i}^{\prime}(i=1,2, \cdots, j) \quad$ with $\quad p_{i}^{\prime} p_{i}<\delta \quad(i=1,2, \cdots, j)$ and $l\left(T\left(E^{\prime}\right)\right) \leqq$ $l(T(E))-\varepsilon_{0}$. But for $\varepsilon_{0}$, there is $\delta=\varepsilon_{0} /(3(2 n-3))$ such that $p_{i}^{\prime \prime} p_{i}^{\prime}<\delta(i=1,2, \cdots, j)$ implies that $l\left(T\left(E^{\prime \prime}\right)\right)<l\left(T\left(E^{\prime}\right)\right)+\varepsilon_{0}$ (by the first part of the proof). (Here $l\left(T\left(E^{\prime \prime}\right)\right)$ denotes the linear content of a mini-tree on $\left.E^{\prime \prime}=\left\{p_{1}^{\prime \prime}, p_{2}^{\prime \prime}, \cdots, p_{j}^{\prime \prime}, p_{j+1}, \cdots, p_{n}\right\}\right)$. In particular, the $p_{i}^{\prime \prime}=p_{i}(i=1,2, \cdots, j)$ are such that $p_{i}^{\prime \prime} p_{i}^{\prime}<\delta$. Then $l(T(E))<$ $l\left(T\left(E^{\prime}\right)\right)+\varepsilon_{0} \leqq\left(l(T(E))-\varepsilon_{0}\right)+\varepsilon_{0}=l(T(E))$. This contradiction establishes the result.

Proof of Theorem 2. Let $P$ be a finite normally ordered subset of a rectifiable arc $A$ of a finitely compact metric space $M$ and $l(T(P))$ denote the linear content of a mini-tree on $P$ (and spanning vertex set $\left.P^{*} \supset P\right)$. Defining $l^{*}(A)=$ l.u.b. . $_{P A A} l(T(P))$, it suffices to show that $l^{*}(A) \geqq l(A)$. To do this let $l(A)=$ l.u.b. $p \subset A$ $\sum_{i=1}^{n-1} p_{i} p_{i+1}$ and denote by $A_{i}$ the subarc of $A$ lying between $p_{i}$ and $p_{i+1}$. Then $l^{*}\left(A_{i}\right) \geqq p_{i} p_{i+1}$ holds, and it remains to show that $l^{*}(A) \geqq \sum_{i=1}^{n-1} l^{*}\left(A_{i}\right)$. But this follows by complete induction on the statement $l^{*}\left(A_{1}+A_{2}\right) \geqq$ $l^{*}\left(A_{1}\right)+l^{*}\left(A_{2}\right)$ the proof of which follows.

For an arbitrary $\varepsilon>0$, there exists two finite subsets $E_{1}^{\prime} \subset A_{1}$ and $E_{2}^{\prime} \subset A_{2}$ such that $l^{*}\left(A_{1}\right)-\varepsilon \leqq l\left(T\left(E_{1}^{\prime}\right)\right)$ and $l^{*}\left(A_{2}\right)-\varepsilon \leqq l\left(T\left(E_{2}^{\prime}\right)\right)$, where $T\left(E_{i}^{\prime}\right)$ denotes a mini-tree on $E_{i}^{\prime}(i=1,2)$. By Theorem $\mathrm{E}$, the linear content $l\left(T\left(E_{i}^{\prime}\right)\right)$ varies an arbitrarily small amount in a sufficiently small neighborhood of a point of $E_{i}^{\prime}$. It is thus possible to choose $E_{1}^{\prime}$ and $E_{2}^{\prime}$ such that the common endpoint $p_{2}$ of $A_{1}$ and $A_{2}$ lies neither in $E_{1}^{\prime}$ nor $E_{2}^{\prime}$, so the last point $q_{1}$ of $E_{1}^{\prime}$ and the first point $q_{2}$ of $E_{2}^{\prime}$ are different from $p_{2}$. Let $A_{1}^{\prime}$ be the subarc of $A$ between $p_{1}$ and $q_{1} ; A_{2}^{\prime}$ the subarc between $q_{2}$ and $p_{3}$. 
Since a metric space is a normal topological space, there exist two open sets $U_{1}$ and $U_{2}$ which contain the subarcs $A_{1}^{\prime}$ and $A_{2}^{\prime}$, respectively, and which have disjoint closures. If $d$ denotes the distance of the set $A_{1}^{\prime}+A_{2}^{\prime}$ from the boundary of $U_{1}+U_{2}$, then $d>0$. Let $\delta$ be such that $0<\delta<\min \left\{d, \varepsilon d / l^{*}\left(A_{1}+A_{2}\right)\right\}$. There exist two normally ordered finite sets $E_{1}$ and $E_{2}$ such that $E_{1}^{\prime} \subset$ $E_{1} \subset A_{1}^{\prime}$ and $E_{2}^{\prime} \subset E_{2} \subset A_{2}^{\prime}$, and such that each two successive points in $E_{1}$ and $E_{2}$ have distance less than $\delta$. Let $E=E_{1}+E_{2}$ and let $l(T(E))$ denote the linear content of a mini-tree on $E$ (and having vertex set $\left.E^{*} \supset E\right)$.

Now decompose the finite sets $E_{i}(i=1,2)$ into finitely many classes such that two points of $E_{i}$ are in the same class provided the set $E^{*} \cdot \bar{U}_{i}$ contains the end points of segments of a chain of $T(E)$ joining the two points. If a given point of $E_{i}$ is not connected by such a chain to any other point of $E_{i}$, then the point is in a class by itself. Let $n_{i}(i=1,2)$ be the number of these classes. Since $E^{*}$ is connected by chains of $T(E)$, every two classes of $E_{i}$ are such that each point of one class is joined by a chain of $T(E)$ to each point of the other, but which does not have all of its vertices contained in $\bar{U}_{i}$. According tothe definition of these classes and because of the disjointness of $\bar{U}_{1}$ and $\bar{U}_{2}$, every two of these $n_{1}+n_{2}$ chains are disjoint. On the other hand, each pair of these chains has distance apart at least $d$, since $E_{1}+E_{2}$ as a subset of $A_{1}^{\prime}+A_{2}^{\prime}$ has distance $\geqq d$ from the boundary of $U_{1}+U_{2}$. Then $\left(n_{1}+n_{2}\right) d \leqq$ $l(T(E)) \leqq l^{*}\left(A_{1}+A_{2}\right)$, and so $\delta<\varepsilon d /\left(\left(n_{1}+n_{2}\right) d\right)=\varepsilon /\left(n_{1}+n_{2}\right)$. Hence $\left(n_{1}+n_{2}\right) \delta<\varepsilon$.

Now for each class, except for the two which contain the last points of $E_{1}$ and $E_{2}$, introduce a segment consisting of the last point of the class and the next point of $E_{1}$ or $E_{2}$ (according as the class is in $E_{1}$ or $E_{2}$, respectively). Since each pair of successive points in $E_{1}$ and $E_{2}$ has distance $\leqq \delta$, then each of the above $n_{1}+n_{2}-2$ segments has length $\leqq \delta$ and is therefore contained in $\bar{U}_{1}$ or $\bar{U}_{2}$, respectively. Augmenting $T(E)$ with these $n_{1}+n_{2}-2$ segments yields a set $S$ of segments containing $T(E)$ and such that $l(S) \leqq$ $l(T(E))+\left(n_{1}+n_{2}-2\right) \cdot \delta<l(T(E))+\left(n_{1}+n_{2}\right) \cdot \delta<l(T(E))+\varepsilon$.

Set $S$ is such that each two points in $E_{i}$ are joined by a unique chain (of $S$ ) which is contained entirely in $\bar{U}_{i}$. Since $\bar{U}_{1}$ and $\bar{U}_{2}$ are disjoint, $S$ must contain two disjoint trees $T_{1}\left(E_{1}\right)$ on $E_{1}$ and $T_{2}\left(E_{2}\right)$ on $E_{2}$, such that $l\left(T_{1}\left(E_{1}\right)\right)+l\left(T_{2}\left(E_{2}\right)\right) \leqq l(S)<l(T(E))+\varepsilon$. Therefore, for mini-trees $T\left(E_{1}\right)$ and $T\left(E_{2}\right)$ on $E_{1}$ and $E_{2}$, respectively, $l\left(T\left(E_{1}\right)\right)+$ $l\left(T\left(E_{2}\right)\right)<l(T(E))+\varepsilon$.

On the other hand, $E_{i}^{\prime} \subset E_{i}$ implies that $l\left(T\left(E_{i}^{\prime}\right)\right) \leqq l\left(T\left(E_{i}\right)\right)$ and since $E_{i}^{\prime}$ was chosen so that $l^{*}\left(A_{i}\right)-\varepsilon \leqq l\left(T\left(E_{i}^{\prime}\right)\right)(i=1,2)$, 


$$
\begin{aligned}
l^{*}\left(A_{1}\right)+l^{*}\left(A_{2}\right) & \leqq l\left(T\left(E_{1}^{\prime}\right)\right)+\varepsilon+l\left(T\left(E_{2}^{\prime}\right)\right)+\varepsilon \\
& \leqq l\left(T\left(E_{1}\right)\right)+l\left(T\left(E_{2}\right)\right)+2 \varepsilon \\
& <(l(T(E))+\varepsilon)+2 \varepsilon \\
& \leqq \sup l(T(E))+3 \varepsilon \\
& =l^{*}\left(A_{1}+A_{2}\right)+3 \varepsilon .
\end{aligned}
$$

Since this holds for each $\varepsilon>0, l^{*}\left(A_{1}\right)+l^{*}\left(A_{2}\right) \leqq l^{*}\left(A_{1}+A_{2}\right)$, and the theorem is proved.

\section{REFERENCES}

1. L. M. Blumenthal, Theory and Applications of Distance Geometry. London: Oxford University Press, 1953.

2. E. J. Cockayne, On the Steiner problem, Cand. Math. Bull., 10, Number 3, (1967).

3. K. Menger and Mimura, Ergebnisse eines mathematischen Kolloquims, Wien, Heft, 4 (1932).

Received December 20, 1976 and in revised form December 2, 1977.

Southeast Missouri State University

Cape Girardeau, MO 63701 


\section{PACIFIC JOURNAL OF MATHEMATICS}

\section{EDITORS}

RICHARD ARENS (Managing Editor)

University of California

Los Angeles, CA 90024

Charles W. Curtis

University of Oregon

Eugene, OR 97403

C. C. MOORE

University of California

Berkeley, CA 94720

\section{J. DugundJI}

Department of Mathematics

University of Southern California

Los Angeles, CA 90007

R. FinN and J. Milgram

Stanford University

Stanford, CA 94305

\section{ASSOCIATE EDITORS}
E. F. BECKENBACH
B. H. NeumanN
F. WOLF
K. YosHIDA

\section{SUPPORTING INSTITUTIONS}

\author{
UNIVERSITY OF BRITISH COLUMBIA \\ CALIFORNIA INSTITUTE OF TECHNOLOGY \\ UNIVERSITY OF CALIFORNIA \\ MONTANA STATE UNIVERSITY \\ UNIVERSITY OF NEVADA, RENO \\ NEW MEXICO STATE UNIVERSITY \\ OREGON STATE UNIVERSITY \\ UNIVERSITY OF OREGON
}

\author{
UNIVERSITY OF SOUTHERN CALIFORNIA \\ STANFORD UNIVERSITY \\ UNIVERSITY OF HAWAII \\ UNIVERSITY OF TOKYO \\ UNIVERSITY OF UTAH \\ WASHINGTON STATE UNIVERSITY \\ UNIVERSITY OF WASHINGTON
}

The Supporting Institutions listed above contribute to the cost of publication of this Journal, but they are not owners or publishers and have no responsibility for its content or policies.

Mathematical papers intended for publication in the Pacific Journal of Mathematics should be in typed form or offset-reproduced, (not dittoed), double spaced with large margins. Please do not use built up fractions in the text of the manuscript. However, you may use them in the displayed equations. Underline Greek letters in red, German in green, and script in blue. The first paragraph or two must be capable of being used separately as a synopsis of the entire paper. Items of the bibliography should not be cited there unless absolutely necessary, in which case they must be identified by author and journal, rather than by item number. Manuscripts, in triplicate, may be sent to any one of the editors. Please classify according to the scheme of Math. Reviews, Index to Vol. 39. All other communications should be addressed to the managing editor, or Elaine Barth, University of California, Los Angeles, California, 90024.

50 reprints to each author are provided free for each article, only if page charges have been substantially paid. Additional copies may be obtained at cost in multiples of 50 .

The Pacific Journal of Mathematics is issued monthly as of January 1966. Regular subscription rate: $\$ 72.00$ a year (6 Vols., 12 issues). Special rate: $\$ 36.00$ a year to individual members of supporting institutions.

Subscriptions, orders for numbers issued in the last three calendar years, and changes of address should be sent to Pacific Journal of Mathematics, 103 Highland Boulevard, Berkeley, California, 94708. Older back numbers obtainable from Kraus Periodicals Co., Route 100, Millwood, NY 10546.

PUBLISHED BY PACIFIC JOURNAL OF MATHEMATICS, A NON-PROFIT CORPORATION

Printed at Kokusai Bunken Insatsusha (International Academic Printing Co., Ltd.). 8-8, 3-chome, Takadanobaba, Shinjuku-ku, Tokyo 160, Japan.

Copyright (C) 1978 by Pacific Journal of Mathematics Manufactured and first issued in Japan 


\section{Pacific Journal of Mathematics}

\section{Vol. 76, No. $2 \quad$ December, 1978}

Stephanie Brewster Brewer Taylor Alexander, Local and global convexity in complete Riemannian manifolds ...........................

Claudi Alsina i Català, On countable products and algebraic convexifications of probabilistic metric spaces ...............................

Joel David Berman and George Grätzer, Uniform representations of

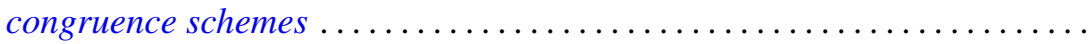

Ajit Kaur Chilana and Kenneth Allen Ross, Spectral synthesis in

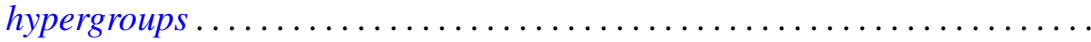

David Mordecai Cohen and Howard Leonard Resnikoff, Hermitian quadratic forms and Hermitian modular forms . .........................

Frank Rimi DeMeyer, Metabelian groups with an irreducible projective

representation of large degree .............................

Robert Ellis, The Furstenberg structure theorem .....................

Heinz W. Engl, Random fixed point theorems for multivalued mappings .......

William Andrew Ettling, On arc length sharpenings ..................

Kent Ralph Fuller and Joel K. Haack, Rings with quivers that are trees........

Kenneth R. Goodearl, Centers of regular self-injective rings ...............

John Gregory, Numerical algorithms for oscillation vectors of second order

differential equations including the Euler-Lagrange equation for

symmetric tridiagonal matrices.

Branko Grünbaum and Geoffrey Shephard, Isotoxal tilings

Myron Stanley Henry and Kenneth Leroy Wiggins, Applications of

approximation theory to differential equations with deviating

arguments

Mark Jungerman, The non-orientable genus of the n-cube .

Robert Richard Kallman, Only trivial Borel measures on $S_{\infty}$ are

quasi-invariant under automorphisms ................

Joyce Longman and Michael Rich, Scalar dependent algebras in the

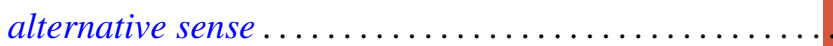

Richard A. Mollin, The Schur group of a field of characteristic zero ........ 471

David Pokrass, Some radical properties of rings with $(a, b, c)=(c, a, b) \ldots 479$

Margaret Shay and Paul Ruel Young, Characterizing the orders changed by

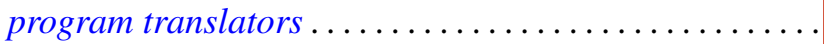

Jerrold Norman Siegel, On the structure of $B_{\infty}(F), F$ a stable space...

Surjeet Singh, (hnp)-rings over which every module admits a basic

submodule...

A. K. Snyder, Universal interpolating sets and the Nevanlinna-Pick property in

Banach spaces of functions...

Jeffrey D. Vaaler, On the metric theory of Diophantine approximation ... 